\title{
Dietary intake, glucose metabolism and sex hormones in women with polycystic ovary syndrome (PCOS) compared with women with non-PCOS-related infertility
}

\author{
Ya-Hui Tsai ${ }^{1} \dagger$, Ting-Wen Wang ${ }^{1} \dagger$, Hsiao-Jui Wei ${ }^{2}$, Chien-Yeh Hsu ${ }^{3}$, Hsin-Jung Ho ${ }^{1}$, \\ Wen-Hua Chen ${ }^{3}$, Robert Young ${ }^{2}$, Chian-Mey Liaw ${ }^{2}$ and Jane C.-J. Chao ${ }^{1,4 *}$ \\ ${ }^{1}$ School of Nutrition and Health Sciences, Taipei Medical University, Taipei, Taiwan, ROC \\ ${ }^{2}$ Infertility Center, Taiwan Adventist Hospital, Taipei, Taiwan, ROC \\ ${ }^{3}$ Graduate Institute of Biomedical Informatics, Taipei Medical University, Taipei, Taiwan, ROC \\ ${ }^{4}$ Nutrition Research Center, Taipei Medical University Hospital, Taipei, Taiwan, ROC
}

(Submitted 18 November 2011 - Final revision received 21 August 2012 - Accepted 22 August 2012 - First published online 10 October 2012)

\section{Abstract}

The present study investigated dietary intake, glucose metabolism and sex hormones in women with polycystic ovary syndrome (PCOS). A total of forty-five women (aged 25-40 years) with PCOS and 161 control women (aged 25-43 years) with non-PCOS-related infertility were recruited. Anthropometry, glucose tolerance and sex hormones were determined and dietary intake was assessed. Women with PCOS had lower serum sex hormone-binding globulin and increased BMI, waist:hip ratio, luteinising hormone, ratio of luteinising hormone: follicle-stimulating hormone, testosterone and free androgen index (FAI). Postprandial glucose, fasting insulin and insulin resistance were elevated in women with PCOS. Women with PCOS had reduced energy and carbohydrate intake but higher fat intake. Serum sex hormone-binding globulin level was negatively associated with BMI in both groups and negatively correlated with macronutrient intake in the PCOS group with hyperandrogenism. However, FAI was positively correlated with BMI, waist circumference and glucose metabolic parameters in both groups. Therefore, women with PCOS consume lower energy and carbohydrate compared with those with non-PCOS-related infertility and macronutrient intake is only negatively associated with serum sex hormone-binding globulin level in the PCOS group with hyperandrogenism.

Key words: Polycystic ovary syndrome: Dietary intake: Glucose metabolism: Sex hormones

Polycystic ovary syndrome (PCOS), characterised by oligoand/or anovulation, clinical and/or biochemical signs of hyperandrogenism and/or polycystic ovaries ${ }^{(1)}$, is one of the most common causes of ovulation dysfunction in women of reproductive age. The prevalence of PCOS has been found to range from 2 to $20 \%$ in the general population worldwide $^{(2)}$, varying depending on the criteria used. According to the National Institutes of Health criteria (1990), Rotterdam consensus criteria (2003) and Androgen Excess Society criteria (2006), the estimated prevalence of PCOS was 8.7, 11.9-17.8 and $10 \cdot 2-12 \cdot 0 \%$, respectively, in Australia $^{(3)}$. PCOS can be suspected by clinical and laboratory findings including irregular menstruation or amenorrhea, hirsutism, acne and/or enlarged polycystic ovaries in women with infertility, but excludes other androgen excess or ovulatory disorders, such as congenital adrenal hyperplasia androgen-secreting neoplasms, Cushing's syndrome, thyroid dysfunction, severe insulin resistance, hyperprolactinaemia, or exogenous androgen excess ${ }^{(1,4)}$. In addition to abnormal clinical and biochemical signs of hyperandrogenism, insulin resistance, compensatory hyperinsulinaemia and impaired glucose tolerance could increase the risk of type 2 diabetes mellitus and hyperlipidaemia in women with $\operatorname{PCOS}^{(5,6)}$.

Obesity is common in $30-70 \%$ women with $\operatorname{PCOS}^{(6)}$. Obesity, in particular of the abdominal type, is present in variable degrees in women with PCOS studied in Europid populations ${ }^{(7,8)}$. However, there are a lower prevalence rate of glucose intolerance and lower BMI in Chinese women with $\operatorname{PCOS}^{(9)}$. Obesity plays a crucial role in the pathogenesis of PCOS when accompanied with insulin resistance ${ }^{(10)}$. About $50-70 \%$ women with PCOS have insulin resistance and secondary hyperinsulinaemia ${ }^{(11)}$. Insulin resistance has been

Abbreviations: FAI, free androgen index; FSH, follicle-stimulating hormone; HOMA-IR, homeostasis model assessment of insulin resistance; LH, luteinising hormone; PCOS, polycystic ovary syndrome; SHBG, sex hormone-binding globulin; WHR, waist:hip ratio.

*Corresponding authors: ; Dr Jane C.-J. Chao, fax + 88622737 3112, email chenjui@tmu.edu.tw; Dr Hsiao-Jui Wei, email wei0937059468@yahoo.com.tw † Joint first authors. 
reported in non-obese Chinese women with $\operatorname{PCOS}^{(12)}$, indicating that the presence of hyperinsulinaemia in women with PCOS is independent of obesity. Insulin resistance is associated with an increased risk of the metabolic syndrome, diabetes mellitus and $\mathrm{CVD}^{(13)}$. It is hypothesised that controlled body weight and improved insulin resistance may delay or prevent the development of diabetes mellitus and CVD in women with PCOS $^{(6)}$.

Several studies have revealed that weight loss by diet, exercise and/or behaviour intervention improves body composition, insulin resistance and hyperandrogenism as well as restores menstrual cyclicity and ovulation in overweight or obese women with $\operatorname{PCOS}^{(14-16)}$. A low-glycaemic index diet has been reported to improve menstrual cyclicity in overweight and obese women with $\operatorname{PCOS}^{(16)}$. However, central obesity and insulin resistance were not strictly correlated with dietary intake including energy and macronutrients in women with $\operatorname{PCOS}^{(14)}$. It is unclear whether dietary intake, glucose metabolism and sex hormones in women with PCOS differ from those with non-PCOS-related infertility and whether the changes in sex hormones are associated with anthropometric measurements, glucose metabolism and/or dietary intake in women with PCOS. Therefore, the present study investigated dietary intake, glucose metabolism and sex hormones in women with PCOS and the correlation between sex hormones and other variables, such as anthropometric measurements, glucose metabolism and dietary intake.

\section{Methods}

\section{Subjects}

A total of forty-five women with PCOS (aged 25-40 years) and 161 non-PCOS infertile women of a similar age (25-43 years) as controls were recruited from 287 patients who visited the Infertility Center of Taiwan Adventist Hospital for the first time between April 2008 and March 2009. Aetiologies of infertility in the subjects were: $37 \cdot 7 \%$ ovulation dysfunction (including women with PCOS), 9.5\% male factor, $8.6 \%$ endometriosis, $1.4 \%$ tubal factor, $1.1 \%$ poor ovarian function, $15.0 \%$ multiple factors and $26.7 \%$ unexplained causes. The diagnosis of PCOS was based on revised 2003 Rotterdam criteria established by the European Society for Human Reproduction and Embryology and American Society for Reproductive Medicine ${ }^{(1)}$. The subjects with non-classic adrenal hyperplasia, 21-hydroxylase deficiency, hyperprolactinaemia, hypothyroidism, androgen-secreting tumours and receiving hormonal therapy were excluded from the present study. The present study was conducted according to the guidelines laid down in the Declaration of Helsinki and all procedures involving human subjects were approved by the Taiwan Adventist Hospital Institutional Review Board. Written informed consent was obtained from all subjects before the study.

\section{Protocols}

The subjects completed the standard form for medical history including menstrual history and anthropometric measurements including height, body weight, BMI, waist circumference and waist:hip ratio (WHR). An overnight fasting blood sample was obtained from the subjects between 08.00 and 10.00 hours during the first $3 \mathrm{~d}$ of the menstrual cycle for hormone assays including follicle-stimulating hormone (FSH), luteinising hormone (LH), testosterone and sex hormone-binding globulin (SHBG). Blood samples were stored at $-80^{\circ} \mathrm{C}$ until analysis within $7 \mathrm{~d}$. Blood glucose and insulin levels were measured before and $2 \mathrm{~h}$ after a $75 \mathrm{~g}$ oral glucose load in $300 \mathrm{ml}$ water. Dietary intake was assessed by 3-d dietary record.

\section{Laboratory assays}

Serum FSH, LH, testosterone and SHBG were assessed by commercial RIA kits (Diagnosis System Laboratories, Inc.) Free androgen index (FAI), an indicator of an abnormal androgen status in women, was determined by:

$$
\mathrm{FAI}=\text { total testosterone }(\mathrm{nmol} / \mathrm{l}) / \text { SHBG }(\mathrm{nmol} / \mathrm{l}) \times 100 \text {. }
$$

Blood glucose concentration was determined by the glucose oxidase method $30 \mathrm{~min}$ after blood was drawn. Serum insulin was analysed by a commercial RIA kit (Diagnosis Products Corp.). Homeostasis model assessment of insulin resistance (HOMA-IR $)^{(17)}$, an index of insulin resistance, was calculated as:

$$
\begin{aligned}
\text { HOMA }-\mathrm{IR}= & \text { fasting glucose }(\mathrm{mmol} / \mathrm{l}) \\
& \times \text { fasting insulin }(\mu \mathrm{U} / \mathrm{ml}) / 22 \cdot 5 .
\end{aligned}
$$

\section{Dietary assessment}

The subjects were taught food portion sizes using food photographs and measuring containers and given instructions for the dietary record by a registered dietitian before recording dietary intake. A 3-d dietary record of their usual diets including two weekdays and one weekend was completed by the subjects in the week after blood was drawn. The registered dietitian re-checked mealtime, eating place, food items or ingredients, cooking methods and portion sizes by telephone interview after the dietary record sheet was returned. Energy and macronutrient intake was analysed using Food and Nutrition Data Analysis System for Research software (Ekitchen Business Co.). Dietary intake data were removed because of under-reporting of energy intake, which was defined as below $70 \%$ of dietary reference intake in Taiwan.

\section{Statistical analysis}

All data are expressed as mean values and standard deviations. Statistical analysis was performed using SAS (version 9.1; SAS Institute Inc.). The normality of the data was tested by the Kolmogorov-Smirnov test. In order to determine the effect of BMI and hyperandrogenism on clinical measurements, the subjects in the PCOS and control groups were divided into two subgroups by BMI $\left(<22\right.$ and $\left.\geq 22 \mathrm{~kg} / \mathrm{m}^{2}\right)$ and those in 
the PCOS group were divided into two subgroups by serum testosterone level $(\leq 1.7$ and $>1.7 \mathrm{nmol} / \mathrm{l})$ for further analysis. The BMI cut-off point established in 1995 by the Department of Health in Taiwan for ideal weight was $22 \mathrm{~kg} / \mathrm{m}^{2}$. According to current criteria of the Department of Health in Taiwan, the values of BMI for underweight, normal weight, overweight and obesity are $<18.5,18.5-23.9,24.0-26.9$ and $\geq 27.0 \mathrm{~kg} / \mathrm{m}^{2}$, respectively. The BMI cut-off point for the subgroups was set at $22 \mathrm{~kg} / \mathrm{m}^{2}$, which is the same cut-off point for ideal weight in the present study. Hyperandrogenism was diagnosed biochemically by a serum testosterone level over $1.7 \mathrm{nmol} / \mathrm{l}^{(18)}$, which was set for the cut-off point. The data among different subgroups in the control and PCOS groups were analysed by one-way ANOVA and Fisher's least significant difference test. The differences between the PCOS and control groups as well as between the two subgroups in women with PCOS were analysed by Student's $t$ test. Correlations between two variables were analysed by simple linear regression and Pearson's correlation coefficient. Multiple linear regression analysis was used for predicting the association of clinical measurements and independent variables, such as characteristics, glucose metabolic parameters and dietary intake. The statistical significance level was set at $P<0.05$.

\section{Results}

\section{Demographic and clinical characteristics}

All participants completed anthropometric and clinical measurements and dietary records in the study. The number of women with normal $(\leq 1.7 \mathrm{nmol} / \mathrm{l})$, intermediate $(1 \cdot 8-3 \cdot 1 \mathrm{nmol} / \mathrm{l})$ and high $(>3 \cdot 1 \mathrm{nmol} / \mathrm{l})$ serum testosterone level in the PCOS group was twenty-four (53\%), eighteen ( $40 \%)$ and three $(7 \%)$, respectively. Of the women, eight controls (5\%) and six with PCOS (13\%) were obese, with BMI $\geq 27 \mathrm{~kg} / \mathrm{m}^{2}$ defined as obesity in Taiwan, and five controls (3\%) and four with PCOS (9\%) had BMI $>30 \mathrm{~kg} / \mathrm{m}^{2}$. Body weight $(P=0.009)$, BMI $(P=0.033)$, waist circumference $(P=0.005)$ and WHR $(P=0.039)$ were significantly higher in the PCOS group than those in the control group (Table 1).

The PCOS group had significantly increased serum LH $(P<0.0001)$, LH:FSH $(P<0.0001)$, testosterone $(P=0.036)$ and FAI $(P<0.0001)$, but lowered serum SHBG $(P=0.002)$ compared with the control group (Table 1$)$. The control group with BMI $\geq 22 \mathrm{~kg} / \mathrm{m}^{2}$ had significantly decreased serum FSH level $(P=0.033)$ compared with those with BMI $<22 \mathrm{~kg} / \mathrm{m}^{2}$. Both control and PCOS groups with BMI $\geq 22 \mathrm{~kg} / \mathrm{m}^{2}$ had significantly decreased serum SHBG levels $(P<0.0001$ and $P=0.033$ for the control and PCOS groups) compared with the corresponding groups with BMI $<22 \mathrm{~kg} / \mathrm{m}^{2}$. The PCOS group with BMI $\geq 22 \mathrm{~kg} / \mathrm{m}^{2}$ had elevated serum LH $(P=0.0005)$, LH:FSH $(P=0.009)$ and FAI $(P<0 \cdot 0001)$ compared with the control group with BMI $\geq 22 \mathrm{~kg} / \mathrm{m}^{2}$.

The PCOS group had significantly elevated 2 -h serum glucose $(P=0 \cdot 048)$, fasting insulin $(P=0.004)$ and HOMA-IR ( $P=0.004)$ compared with the control group (Table 1$)$. The control group with $\mathrm{BMI} \geq 22 \mathrm{~kg} / \mathrm{m}^{2}$ had increased fasting glucose $(P=0 \cdot 004), 2$-h serum glucose $(P=0 \cdot 003)$, fasting insulin $(P=0.002), 2$-h serum insulin $(P=0.001)$ and HOMA-IR $(P=0.001)$ compared with those with BMI $<22 \mathrm{~kg} / \mathrm{m}^{2}$. The PCOS group with BMI $\geq 22 \mathrm{~kg} / \mathrm{m}^{2}$ had significantly elevated 2-h serum glucose $(P=0 \cdot 002)$, fasting insulin $(P=0 \cdot 015), 2$-h

Table 1. Demographic and clinical characteristics of the control and polycystic ovary syndrome (PCOS) by BMI category (Mean values and standard deviations)

\begin{tabular}{|c|c|c|c|c|c|c|c|c|c|c|c|c|}
\hline \multirow[t]{3}{*}{ Group... } & \multicolumn{6}{|c|}{ Control } & \multicolumn{6}{|c|}{ PCOS } \\
\hline & \multicolumn{2}{|c|}{$\begin{array}{c}\mathrm{BMI}<22 \mathrm{~kg} / \mathrm{m}^{2} \\
\quad(n 112)\end{array}$} & \multicolumn{2}{|c|}{$\begin{array}{c}\mathrm{BMI} \geq 22 \mathrm{~kg} / \mathrm{m}^{2} \\
(n 49)\end{array}$} & \multicolumn{2}{|c|}{ Total $(n 161)$} & \multicolumn{2}{|c|}{$\begin{array}{c}\mathrm{BMI}<22 \mathrm{~kg} / \mathrm{m}^{2} \\
(n 21)\end{array}$} & \multicolumn{2}{|c|}{$\begin{aligned} \mathrm{BMI} & \geq 22 \mathrm{~kg} / \mathrm{m}^{2} \\
& (\text { n } 24)\end{aligned}$} & \multicolumn{2}{|c|}{ Total $(n 45)$} \\
\hline & Mean & SD & Mean & SD & Mean & SD & Mean & SD & Mean & SD & Mean & SD \\
\hline Age (years) & 35.5 & 3.4 & $34 \cdot 8$ & 4.0 & $34 \cdot 7$ & 3.6 & $32 \cdot 3 \dagger$ & $2 \cdot 8$ & $33 \cdot 0$ & $5 \cdot 2$ & $32 \cdot 7$ & 4.2 \\
\hline Weight (kg) & $51 \cdot 2$ & 4.6 & $61 \cdot 8^{*}$ & 7.5 & 54.4 & 7.4 & $50 \cdot 9$ & 4.5 & $66 \cdot 5^{\star}$ & $11 \cdot 3$ & $59 \cdot 2 \ddagger$ & $11 \cdot 8$ \\
\hline BMI $\left(\mathrm{kg} / \mathrm{m}^{2}\right)$ & 19.9 & 1.3 & $24 \cdot 4^{*}$ & $3 \cdot 1$ & $21 \cdot 3$ & 2.9 & $19 \cdot 6$ & $1 \cdot 2$ & $26 \cdot 0^{*} \dagger$ & 4.0 & $23.0 \ddagger$ & 4.4 \\
\hline Waist $(\mathrm{cm})$ & $66 \cdot 6$ & 3.6 & $75 \cdot 5^{\star}$ & $6 \cdot 8$ & $69 \cdot 3$ & $6 \cdot 3$ & $66 \cdot 8$ & $5 \cdot 7$ & $78 \cdot 3^{*} \dagger$ & $11 \cdot 0$ & $72 \cdot 9 \ddagger$ & $10 \cdot 6$ \\
\hline WHR & 0.72 & 0.05 & $0.76^{\star}$ & 0.06 & 0.74 & 0.05 & 0.73 & 0.05 & $0 \cdot 76^{*}$ & 0.08 & $0.75 \ddagger$ & 0.07 \\
\hline FSH (IU/I) & $7 \cdot 8$ & $2 \cdot 8$ & $6 \cdot 9^{*}$ & 1.9 & 7.5 & $2 \cdot 6$ & $6 \cdot 9$ & $1 \cdot 7$ & $6 \cdot 7$ & 1.9 & $6 \cdot 8$ & 1.8 \\
\hline LH (IU/I) & 4.8 & $2 \cdot 1$ & $3 \cdot 7$ & $1 \cdot 8$ & 4.5 & $2 \cdot 1$ & $8.2 \dagger$ & $6 \cdot 4$ & $6.5 \dagger$ & $5 \cdot 3$ & $7 \cdot 3 \neq \ddagger$ & $2 \cdot 1$ \\
\hline LH:FSH & 0.7 & 0.3 & 0.6 & 0.4 & 0.6 & 0.4 & $1.3+$ & 1.0 & $1.0 \dagger$ & 0.8 & $1 \cdot 1 \neq \ddagger$ & 0.9 \\
\hline Testosterone $(\mathrm{nmol} / \mathrm{l})$ & 1.6 & 0.5 & 1.6 & 0.5 & 1.6 & 0.5 & $1 \cdot 8$ & 0.7 & 1.8 & 0.7 & $1.8 \ddagger$ & 0.7 \\
\hline SHBG $(\mathrm{nmol} / \mathrm{l})$ & $75 \cdot 8$ & $36 \cdot 1$ & $46 \cdot 2^{*}$ & $18 \cdot 6$ & $66 \cdot 6$ & 34.5 & 63.9 & $24 \cdot 6$ & $36 \cdot 7^{\star}$ & $17 \cdot 0$ & $49.4 \ddagger$ & 24.8 \\
\hline FAl & $2 \cdot 57$ & 1.48 & $3 \cdot 76^{\star}$ & 1.85 & 2.93 & 1.69 & 3.38 & $2 \cdot 55$ & $5 \cdot 78^{\star} \dagger$ & 3.08 & 4.68 㧊 & 3.07 \\
\hline Fasting glucose $(\mathrm{mmol} / \mathrm{l})$ & $5 \cdot 1$ & 0.3 & $5 \cdot 3^{*}$ & 0.4 & $5 \cdot 1$ & 0.4 & $5 \cdot 1$ & 0.3 & $5 \cdot 3$ & 0.5 & $5 \cdot 2$ & 0.4 \\
\hline 2-h glucose $(\mathrm{mmol} / \mathrm{l})$ & $5 \cdot 1$ & $1 \cdot 1$ & $5 \cdot 7^{\star}$ & 1.4 & $5 \cdot 2$ & $1 \cdot 2$ & $5 \cdot 1$ & 0.8 & $6 \cdot 2^{*}$ & $1 \cdot 7$ & $5 \cdot 7 \ddagger$ & 1.4 \\
\hline Fasting insulin (pmol/l) & 37.5 & $19 \cdot 4$ & $50 \cdot 7^{\star}$ & $27 \cdot 1$ & $41 \cdot 0$ & 22.9 & $43 \cdot 8$ & $16 \cdot 0$ & $66 \cdot 0^{*}$ & 44.4 & $53.5 \ddagger$ & $34 \cdot 0$ \\
\hline 2-h insulin (pmol/l) & 203 & 149 & $321^{\star}$ & 268 & 240 & 199 & 219 & 184 & $397^{*}$ & 290 & 306 & 253 \\
\hline HOMA-IR & $1 \cdot 22$ & 0.65 & $1.74^{\star}$ & 0.96 & 1.37 & 0.79 & 1.44 & 0.53 & $2 \cdot 27^{\star}$ & 1.56 & $1.81 \neq$ & 1.20 \\
\hline
\end{tabular}

WHR, waist:hip ratio; FSH, follicle-stimulating hormone; LH, luteinising hormone; SHBG, sex hormone-binding globulin; FAI, free androgen index; HOMA-IR, homeostasis model assessment of insulin resistance.

* Mean value was significantly different from that of the BMI $<22 \mathrm{~kg} / \mathrm{m}^{2}$ subgroup in the same group $(P<0.05$; one-way ANOVA and Fisher's least significant different test). † Mean value was significantly different from that of the same BMI subgroup of the control group $(P<0.05$; one-way ANOVA and Fisher's least significant different test). Mean value was significantly different from that of the control group (total): $\ddagger P<0.05$, $\ddagger \ddagger P<0.0001$ (Student's $t$ test). 
serum insulin $(P=0.009)$ and HOMA-IR $(P=0.007)$ compared with the corresponding group with BMI $<22 \mathrm{~kg} / \mathrm{m}^{2}$.

Comparing the two subgroups with serum testosterone level $\leq 1.7$ or $>1.7 \mathrm{nmol} / 1$, the PCOS group with the mean serum testosterone level of $2 \cdot 4 \mathrm{nmol} / 1$ had significantly higher serum LH level $(P=0 \cdot 012)$, LH:FSH $(P=0 \cdot 008)$ and FAI $(P=0.003)$ than the group with the mean serum testosterone level of $1.3 \mathrm{nmol} / 1$ (Table 2). However, there were no significant differences in anthropometric measurements, FSH, SHBG, glucose metabolism and dietary intake between two subgroups with serum testosterone level $\leq 1.7$ or $>1.7 \mathrm{nmol} / 1$ in women with PCOS.

\section{Dietary intake}

The PCOS group had significantly lower daily intakes of energy (6311 (SD 1408) v. $6766(\mathrm{SD} 1080) \mathrm{kJ} / \mathrm{d} ; \quad P=0.002)$ (Fig. 1(a)) and carbohydrate (191 (SD 50) v. 222 (SD 45) g/d, $P<0.0001 ; 51.5$ (SD 8.9) v. 55.0 (SD 6.0)\% of energy, $P<0.0001$ ) (Fig. 1(b) and (c)), but a higher fat intake (30.8 (SD 7.9) v. $28 \cdot 3$ (SD 5.1) \% of energy; $P=0 \cdot 0003$ ) (Fig. 1(c)) than the control group. However, fat intake in terms of $\mathrm{g} / \mathrm{d}$ did not differ between the control and PCOS groups (51 (SD 11) v. $53(\mathrm{SD} \mathrm{17)} \mathrm{g} / \mathrm{d})$. Protein intake in the control and PCOS groups (68 (SD 17) v. 67 (SD 19) g/d; 17.3 (SD 7.3) v. $17 \cdot 6$ (SD $4 \cdot 2) \%$ of energy) did not differ.

Table 2. Demographic and clinical characteristics of the polycystic ovary syndrome group $(n 45)$ by serum testosterone level

(Mean values and standard deviations)

\begin{tabular}{|c|c|c|c|c|}
\hline & \multicolumn{2}{|c|}{$\begin{array}{c}\text { Testosterone } \\
\leq 1.7 \mathrm{nmol} / \mathrm{l} \\
(n 24)\end{array}$} & \multicolumn{2}{|c|}{$\begin{array}{l}\text { Testosterone } \\
>1.7 \mathrm{nmol} / \mathrm{l} \\
(n 21)\end{array}$} \\
\hline & Mean & SD & Mean & SD \\
\hline Age (years) & $33 \cdot 7$ & 3.9 & 31.5 & 4.3 \\
\hline Weight (kg) & $59 \cdot 1$ & 11.4 & $59 \cdot 3$ & $12 \cdot 8$ \\
\hline BMI $\left(\mathrm{kg} / \mathrm{m}^{2}\right)$ & $23 \cdot 0$ & $4 \cdot 1$ & 23.0 & 4.9 \\
\hline Waist (cm) & $73 \cdot 3$ & $10 \cdot 2$ & $72 \cdot 4$ & 11.5 \\
\hline WHR & 0.8 & $0 \cdot 1$ & 0.7 & $0 \cdot 1$ \\
\hline FSH (IU/I) & $7 \cdot 0$ & 1.5 & $6 \cdot 6$ & $2 \cdot 1$ \\
\hline LH (IU/I) & $5 \cdot 1$ & $3 \cdot 2$ & $9 \cdot 8^{*}$ & $7 \cdot 2$ \\
\hline LH:FSH & 0.8 & 0.6 & $1.6^{*}$ & $1 \cdot 1$ \\
\hline Testosterone (nmol/l) & 1.3 & 0.2 & $2 \cdot 4^{\star \star}$ & 0.5 \\
\hline SHBG $(\mathrm{nmol} / \mathrm{l})$ & 51.4 & $28 \cdot 8$ & $47 \cdot 6$ & $20 \cdot 1$ \\
\hline FAl & 3.5 & 2.5 & $6 \cdot 2^{*}$ & $3 \cdot 2$ \\
\hline Fasting glucose $(\mathrm{mmol} / \mathrm{l})$ & $5 \cdot 2$ & 0.4 & $5 \cdot 1$ & 0.3 \\
\hline 2-h glucose $(\mathrm{mmol} / \mathrm{l})$ & $5 \cdot 6$ & 1.3 & $5 \cdot 7$ & 1.6 \\
\hline Fasting insulin (pmol/l) & $52 \cdot 0$ & $30 \cdot 9$ & 57.9 & 40.5 \\
\hline 2-h insulin (pmol/l) & 267 & 209 & 376 & 305 \\
\hline HOMA-IR & 3.0 & 1.6 & $2 \cdot 9$ & 1.8 \\
\hline Energy $(\mathrm{kJ} / \mathrm{d})$ & 6361 & 1330 & 6482 & 1150 \\
\hline Carbohydrate (g) & 195 & 49 & 196 & 37 \\
\hline Carbohydrate ( $\%$ of energy) & $51 \cdot 3$ & $9 \cdot 1$ & $51 \cdot 2$ & 8.5 \\
\hline Protein (g) & 66 & 20 & 69 & 17 \\
\hline Protein (\% of energy) & $17 \cdot 6$ & $5 \cdot 2$ & $17 \cdot 6$ & $2 \cdot 6$ \\
\hline Fat $(\mathrm{g})$ & 53 & 17 & 55 & 17 \\
\hline Fat (\% of energy) & $31 \cdot 1$ & $8 \cdot 2$ & $31 \cdot 2$ & $7 \cdot 4$ \\
\hline
\end{tabular}

WHR, waist:hip ratio; FSH, follicle-stimulating hormone; $\mathrm{LH}$, luteinising hormone; SHBG, sex hormone-binding globulin; FAI, free androgen index; HOMA-IR, homeostasis model assessment of insulin resistance.

Mean value was significantly different from that of the testosterone $\leq 1.7 \mathrm{nmol} / \mathrm{l}$ subgroup: * $P<0.05,{ }^{\star *} P<0.0001$ (Student's $t$ test).
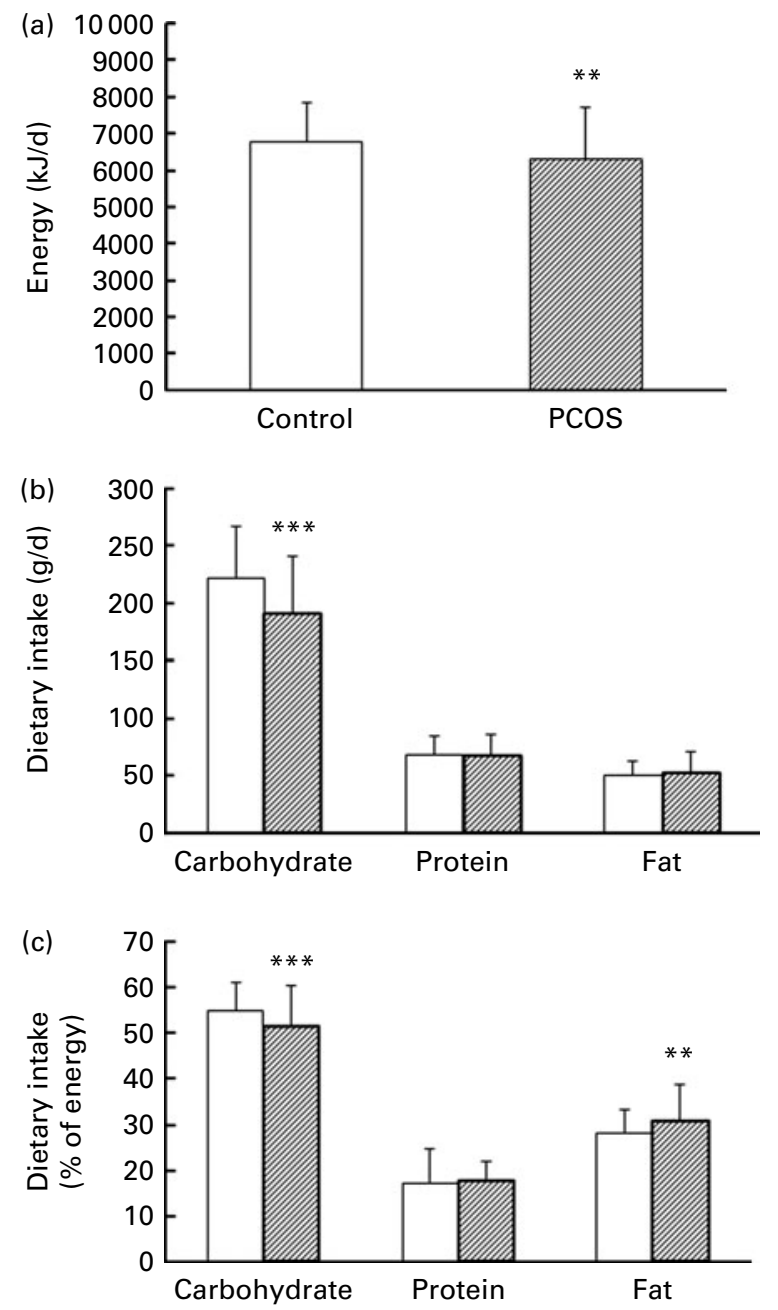

Fig. 1. Daily dietary intake for (a) total energy, (b) macronutrients (g/d) and (c) macronutrients (\% of energy) in the control ( $n 161 ; \square)$ and polycystic ovary syndrome (PCOS) (n 45; ///) groups. Data are means, with standard deviations represented by vertical bars. Mean value was significantly different from that of the control group: ${ }^{\star \star} P<0.01,{ }^{\star * \star} P<0.0001$ (Student's $t$ test).

\section{Correlations between clinical measurements and anthropometric parameters or dietary intake}

Serum FSH level was negatively associated with body weight ( $r-0 \cdot 19 ; P=0 \cdot 016)$, BMI $(r-0 \cdot 21 ; P=0 \cdot 006)$, fasting insulin $(r-0 \cdot 19 ; P=0.014), 2$-h serum insulin $(r-0.21 ; P=0.007)$ and HOMA-IR $(r-0 \cdot 18 ; P=0 \cdot 022)$, but positively associated with protein intake $(\mathrm{g} / \mathrm{d})(r \quad 0.19 ; P=0.018)$ in the control group (Table 3 ). Serum LH level was negatively correlated with body weight $(r-0 \cdot 16 ; P=0.041)$, BMI $(r-0 \cdot 20$; $P=0.013)$, waist circumference $(r-0.21 ; P=0.009)$ and WHR $(r-0 \cdot 16 ; P=0 \cdot 048)$ in the control group. Serum testosterone level was significantly negatively associated with age $(r-0.25 ; P=0 \cdot 001)$, WHR $(r-0 \cdot 18 ; P=0 \cdot 018)$, energy intake $(r-0.18 ; P=0.024)$ and carbohydrate intake $(\mathrm{g} / \mathrm{d})(r-0.20$; $P=0.013$ ), but positively associated with protein intake (\% of energy) $(r \quad 0.20 ; P=0.010)$ in the control group. Serum SHBG level was negatively correlated with body weight $(r-0.43, P=0.0001 v . r-0.59, P<0.0001)$, BMI $(r-0.43$ $v$. $r-0.59 ; \quad P<0.0001)$, waist circumference $(r-0.42$, 
Table 3. Relationships between sex hormones and characteristics, glucose metabolic parameters, or dietary intake in the control ( $n$ 161) and polycystic ovary syndrome (PCOS; $n$ 45) groups

(Pearson's correlation coefficients $(r)$ )

\begin{tabular}{|c|c|c|c|c|c|c|c|c|c|c|}
\hline & \multicolumn{2}{|c|}{$\mathrm{FSH}(\mathrm{IU} / \mathrm{I})$} & \multicolumn{2}{|c|}{$\mathrm{LH}(\mathrm{IU} / \mathrm{l})$} & \multicolumn{2}{|c|}{$\begin{array}{l}\text { Testosterone } \\
(\mathrm{nmol} / \mathrm{l})\end{array}$} & \multicolumn{2}{|c|}{ SHBG (nmol/l) } & \multicolumn{2}{|c|}{$\mathrm{FAl}$} \\
\hline & Control & PCOS & Control & PCOS & Control & PCOS & Control & PCOS & Control & PCOS \\
\hline Age (years) & 0.19 & 0.23 & 0.05 & -0.10 & $-0.25^{\star \star}$ & -0.35 & -0.01 & 0.08 & -0.15 & -0.41 \\
\hline Weight (kg) & $-0.19^{\star}$ & -0.07 & $-0.16^{\star}$ & -0.15 & 0.03 & 0.09 & $-0.43^{\star \star \star}$ & $-0.59^{* * *}$ & $0.40^{\star * \star}$ & $0.55^{\star \star \star}$ \\
\hline BMI $\left(\mathrm{kg} / \mathrm{m}^{2}\right)$ & $-0.21^{\star *}$ & -0.03 & $-0.20^{\star}$ & -0.11 & -0.01 & 0.06 & $-0.43^{\star \star *}$ & $-0.59^{\star \star \star}$ & $0.38^{* \star *}$ & $0.52^{\star \star \star}$ \\
\hline Waist $(\mathrm{cm})$ & -0.15 & -0.15 & $-0.21^{\star *}$ & -0.15 & -0.10 & 0.05 & $-0.42^{\star * *}$ & $-0.55^{\star \star \star}$ & $0.39^{\star \star *}$ & $0.46^{\star *}$ \\
\hline WHR & -0.02 & -0.20 & $-0.16^{\star}$ & -0.13 & $-0.18^{\star}$ & -0.09 & $-0.22^{*}$ & $-0.37^{\star \star}$ & $0.17^{*}$ & 0.23 \\
\hline Fasting glucose $(\mathrm{mmol} / \mathrm{l})$ & 0.03 & 0.05 & 0.004 & -0.07 & -0.04 & -0.07 & $-0.25^{\star}$ & -0.15 & $0.21^{*}$ & -0.02 \\
\hline 2-h glucose $(\mathrm{mmol} / \mathrm{l})$ & -0.07 & 0.26 & -0.08 & -0.24 & -0.09 & 0.07 & $-0.32^{\star \star \star}$ & $-0.33^{\star}$ & $0.21^{\star *}$ & $0.37^{*}$ \\
\hline Fasting insulin $(\mathrm{pmol} / \mathrm{l})$ & $-0.19^{*}$ & 0.03 & 0.02 & 0.06 & 0.003 & 0.11 & $-0.35^{\star \star}$ & -0.29 & $0.38^{\star \star *}$ & $0.41^{*}$ \\
\hline 2-h insulin (pmol/l) & $-0.21^{\star *}$ & 0.19 & -0.05 & 0.01 & -0.08 & 0.28 & $-0.34^{\star \star \star}$ & $-0.35^{\star}$ & $0.30^{\star \star \star}$ & $0.54^{\star \star \star}$ \\
\hline HOMA-IR & $-0.18^{\star}$ & 0.05 & 0.02 & 0.05 & -0.01 & $0 \cdot 10$ & $-0.36^{\star \star \star}$ & $-0.29^{\star}$ & $0.39^{\star \star \star}$ & $0 \cdot 38^{\star \star}$ \\
\hline Energy $(\mathrm{kJ} / \mathrm{d})$ & 0.13 & 0.15 & 0.05 & -0.03 & $-0.18^{\star}$ & 0.11 & 0.12 & 0.07 & -0.13 & 0.04 \\
\hline Carbohydrate (g) & $0 \cdot 13$ & $0.33^{*}$ & -0.003 & 0.03 & $-0.20^{\star}$ & 0.08 & 0.08 & 0.12 & -0.07 & 0.05 \\
\hline Carbohydrate (\% of energy) & 0.05 & $0.30^{*}$ & -0.05 & 0.14 & -0.08 & -0.04 & -0.04 & 0.09 & 0.07 & $-0 \cdot 19$ \\
\hline Protein (g) & $0.19^{\star}$ & -0.02 & -0.002 & 0.02 & $0 \cdot 13$ & 0.04 & 0.08 & -0.18 & -0.05 & 0.14 \\
\hline Protein (\% of energy) & 0.15 & -0.18 & -0.03 & -0.01 & $0.20^{* *}$ & -0.04 & 0.04 & -0.27 & -0.004 & $0 \cdot 18$ \\
\hline Fat $(\mathrm{g})$ & 0.03 & -0.08 & 0.11 & -0.09 & -0.12 & 0.11 & 0.12 & $0 \cdot 10$ & $-0.15^{\star}$ & 0.08 \\
\hline Fat (\% of energy) & -0.07 & -0.24 & 0.09 & -0.14 & 0.02 & 0.07 & 0.06 & 0.05 & -0.09 & 0.12 \\
\hline
\end{tabular}

FSH, follicle-stimulating hormone; LH, luteinising hormone; SHBG, sex hormone-binding globulin; FAl, free androgen index; WHR, waist:hip ratio; HOMA-IR, homeostasis model assessment of insulin resistance.

${ }^{\star} P<0.05,{ }^{\star *} P<0.01,{ }^{\star \star \star} P<0.001$.

$P<0.0001 v . r-0.55, P=0.0002)$, WHR $(r-0.22, P=0.031 v$. $r-0.37, P=0.006), 2$-h serum glucose $(r-0.32, P<0.0001$ v. $r-0.33, P=0.029), 2$-h serum insulin $(r-0.34, P<0.0001$ $v . r-0.35, P=0.019)$ and HOMA-IR $(r-0.36, P<0.0001 v$. $r-0.29, P=0.049)$ in the control and PCOS groups. However, FAI was positively correlated with body weight $(r 0.40 v .0 .55$; $P<0.0001)$, BMI $(r 0.38, P<0.0001$ v. $r 0.52, P=0.0002)$ and waist circumference $(r 0.39, P<0.0001 v . r 0.46, P=0.001)$, 2-h serum glucose $(r 0 \cdot 21, P=0.008 v \cdot r 0 \cdot 37, P=0 \cdot 013)$, fasting insulin $(r 0 \cdot 38, P<0.0001 v . r 0 \cdot 41, P<0 \cdot 05), 2$-h serum insulin

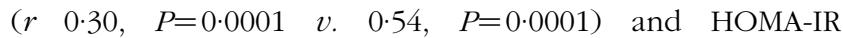
( $r$ 0.39, $P<0.0001$ v. $r 0.38, P=0.009)$ in the control and PCOS groups. FAI was negatively associated with fat intake (g/d) $(r-0.15 ; P=0.048)$ in women with PCOS.
Fasting glucose level was positively correlated with body weight $(r 0.19 ; P=0.018)$ and BMI $(r 0.15 ; P=0.043)$ in the control group (Table 4 ). The levels of 2 -h serum glucose, fasting insulin, 2-h serum insulin and HOMA-IR were positively associated with body weight $(r$ 0.18-0.30, $P=0 \cdot 001-0 \cdot 020$ $v$. $\quad r$ 0.37-0.61, $P<0.0001-0.012)$, BMI (r 0.19-0.33, $P<0.0001-0.01$ v. $r 0.45-0.64, P<0.0001-0.002)$ and waist circumference $(r 0.27-0.34, P<0.0001-0.0006 v . r 0.37-0.55$, $P<0 \cdot 0001-0 \cdot 012)$ in the control and PCOS groups. Fasting insulin level and HOMA-IR were positively correlated with WHR $(r 0.18, P=0.031-0.038 v . r 0.40-0.41$, $P=0.005-0.006)$ in the control and PCOS groups.

Protein intake in terms of $\mathrm{g} / \mathrm{d}$ and percentage of energy was negatively associated with fasting insulin $(r-0 \cdot 19$,

Table 4. Relationships between blood glucose, insulin, or insulin resistance and characteristics or dietary intake in the control ( $n$ 161) and polycystic ovary syndrome (PCOS; $n$ 45) groups

(Pearson's correlation coefficients $(r)$ )

\begin{tabular}{|c|c|c|c|c|c|c|c|c|c|c|}
\hline & \multicolumn{2}{|c|}{$\begin{array}{l}\text { Fasting glucose } \\
\qquad(\mathrm{mmol} / \mathrm{l})\end{array}$} & \multicolumn{2}{|c|}{ 2-h glucose $(\mathrm{mmol} / \mathrm{l})$} & \multicolumn{2}{|c|}{$\begin{array}{l}\text { Fasting insulin } \\
\quad(\mathrm{pmol} / \mathrm{l})\end{array}$} & \multicolumn{2}{|c|}{ 2-h insulin (pmol/l) } & \multicolumn{2}{|c|}{ HOMA-IR } \\
\hline & Control & PCOS & Control & Pcos & Control & PCOS & Control & PCOS & Control & Pcos \\
\hline Age (years) & 0.001 & 0.33 & 0.02 & $0 \cdot 14$ & -0.02 & -0.28 & -0.06 & -0.06 & -0.02 & -0.22 \\
\hline Weight (kg) & $0.19^{\star}$ & 0.12 & $0 \cdot 18^{\star}$ & $0.37^{\star}$ & $0.29^{\star \star \star}$ & $0.61^{\star \star \star}$ & $0.20^{\star}$ & $0.54^{\star \star \star}$ & $0 \cdot 30^{\star \star \star}$ & $0.60^{\star \star \star}$ \\
\hline BMI $\left(\mathrm{kg} / \mathrm{m}^{2}\right)$ & $0 \cdot 15^{\star}$ & $0 \cdot 15$ & $0 \cdot 19^{\star}$ & $0.45^{\star \star}$ & $0.33^{\star \star \star}$ & $0.64^{\star \star \star}$ & $0 \cdot 28^{\star \star \star}$ & $0.60^{\star \star \star}$ & $0.33^{\star \star *}$ & $0.63^{\star \star \star}$ \\
\hline Waist (cm) & $0 \cdot 16$ & $0 \cdot 12$ & $0.27^{\star \star \star}$ & $0 \cdot 37^{\star}$ & $0.33^{\star \star \star}$ & $0.55^{\star \star \star}$ & $0.29^{\star \star \star}$ & $0.45^{\star}$ & $0.34^{\star \star *}$ & $0.53^{\star \star \star}$ \\
\hline WHR & 0.05 & 0.17 & $0.26^{\star \star}$ & 0.30 & $0.18^{\star}$ & $0.41^{\star \star}$ & $0.22^{*}$ & 0.26 & $0.18^{\star}$ & $0.40^{\star *}$ \\
\hline Energy (kJ/d) & -0.06 & -0.02 & -0.15 & -0.03 & -0.03 & -0.04 & -0.12 & 0.01 & -0.04 & -0.02 \\
\hline Carbohydrate (\% of energy) & -0.04 & -0.12 & -0.12 & 0.02 & 0.03 & -0.20 & -0.06 & 0.01 & 0.03 & -0.19 \\
\hline Protein (g) & -0.004 & -0.12 & -0.007 & 0.07 & 0.08 & -0.27 & 0.03 & -0.03 & 0.08 & -0.27 \\
\hline Fat (g) & 0.03 & -0.01 & -0.07 & -0.13 & $-0 \cdot 19^{\star}$ & 0.07 & -0.11 & 0.04 & $-0 \cdot 18^{\star}$ & 0.08 \\
\hline Fat (\% of energy) & 0.08 & 0.003 & -0.002 & -0.12 & $-0.16^{\star}$ & 0.18 & -0.02 & 0.07 & -0.15 & 0.18 \\
\hline Carbohydrate (g) & -0.08 & 0.09 & -0.08 & -0.02 & -0.05 & 0.11 & -0.09 & 0.002 & -0.06 & 0.12 \\
\hline Carbohydrate ( $\%$ of energy) & -0.05 & 0.13 & 0.03 & -0.02 & -0.04 & 0.20 & 0.02 & -0.004 & -0.04 & 0.20 \\
\hline
\end{tabular}

HOMA-IR, homeostasis model assessment of insulin resistance; WHR, waist:hip ratio.

${ }^{\star} P<0.05,{ }^{\star \star} P<0.01,{ }^{\star \star \star} P<0.001$. 
Table 5. Relationships between sex hormones and independent variables including characteristics, glucose metabolic parameters and dietary intake in the control and polycystic ovary syndrome (PCOS) groups using a multiple linear regression model

(Regression coefficients $(\beta)$ )

\begin{tabular}{|c|c|c|c|c|c|c|c|c|}
\hline \multirow[t]{2}{*}{ Group... } & \multicolumn{4}{|c|}{$\begin{array}{l}\text { Control } \\
(n 161)\end{array}$} & \multicolumn{4}{|c|}{$\begin{array}{l}\text { PCOS } \\
(n 45)\end{array}$} \\
\hline & $\begin{array}{l}\text { FSH } \\
(\mathrm{IU} / \mathrm{l})\end{array}$ & $\begin{array}{l}\mathrm{LH} \\
(\mathrm{IU} / \mathrm{I})\end{array}$ & $\begin{array}{l}\text { Testosterone } \\
(\mathrm{nmol} / \mathrm{l})\end{array}$ & $\begin{array}{l}\mathrm{SHBG} \\
(\mathrm{nmol} / \mathrm{l})\end{array}$ & $\begin{array}{l}\text { FSH } \\
(\mathrm{IU} / \mathrm{I})\end{array}$ & $\begin{array}{l}\text { LH } \\
(\mathrm{IU} / \mathrm{I})\end{array}$ & $\begin{array}{l}\text { Testosterone } \\
(\mathrm{nmol} / \mathrm{l})\end{array}$ & $\begin{array}{l}\text { SHBG } \\
(\mathrm{nmol} / \mathrm{l})\end{array}$ \\
\hline \multicolumn{9}{|l|}{ Characteristics } \\
\hline Age (years) & 0.265 & 0.127 & $-0.029^{\star}$ & 0.311 & 0.071 & -0.187 & $-0.089^{\star \star}$ & 0.729 \\
\hline BMI $\left(\mathrm{kg} / \mathrm{m}^{2}\right)$ & 0.084 & -0.002 & 0.003 & $-2 \cdot 764^{\star \star \star}$ & 0.000 & -0.151 & 0.001 & $-3 \cdot 394^{\star \star}$ \\
\hline WHR & -15.031 & -14.325 & $-1 \cdot 175$ & -53.413 & -6.658 & -7.451 & -2.090 & -24.892 \\
\hline \multicolumn{9}{|l|}{ Glucose metabolism } \\
\hline Fasting glucose $(\mathrm{mmol} / \mathrm{l})$ & $2 \cdot 156$ & 1.026 & -0.072 & $-9 \cdot 3861$ & -0.203 & 1.896 & 0.428 & $-7 \cdot 732$ \\
\hline 2-h glucose $(\mathrm{mmol} / \mathrm{l})$ & 0.139 & -0.167 & -0.010 & $-2 \cdot 673$ & 0.330 & -1.800 & -0.114 & -1.852 \\
\hline Fasting insulin (pmol/l) & -0.027 & -0.003 & 0.002 & $-0.279^{*}$ & 0.014 & 0.005 & -0.006 & 0.205 \\
\hline 2-h insulin (pmol//) & -0.002 & -0.001 & -0.000 & -0.017 & -0.001 & 0.008 & $0.002^{*}$ & -0.005 \\
\hline \multicolumn{9}{|l|}{ Dietary intake } \\
\hline Energy (kJ/d) & 0.001 & 0.000 & $-0.0001^{*}$ & 0.002 & 0.000 & -0.000 & -0.000 & -0.001 \\
\hline Carbohydrate (\% of energy) & 73.465 & $20 \cdot 936$ & 2.559 & -399.633 & -21.502 & $190 \cdot 783$ & $-25 \cdot 946$ & $-451 \cdot 217$ \\
\hline Protein (\% of energy) & 68.855 & 21.529 & $4 \cdot 113$ & $-466 \cdot 449$ & $-29 \cdot 243$ & $178 \cdot 322$ & $-27 \cdot 046$ & $-576 \cdot 604$ \\
\hline Fat (\% of energy) & 52.858 & $13 \cdot 105$ & 2.356 & -343.008 & $-27 \cdot 434$ & 175.482 & -25.532 & $-405 \cdot 715$ \\
\hline
\end{tabular}

FSH, follicle-stimulating hormone; LH, luteinising hormone; SHBG, sex hormone-binding globulin; WHR, waist:hip ratio.

${ }^{\star} P<0.05,{ }^{\star \star} P<0.01,{ }^{* \star} P<0.001$.

$P=0.018 v . \quad r-0 \cdot 16, P=0 \cdot 039)$ and HOMA-IR $(r-0 \cdot 18$, $P=0.025 v \cdot r-0 \cdot 15, P=0.052)$ in the control group (Table 4). However, energy and macronutrient intakes were not significantly correlated with glucose metabolic parameters in women with PCOS.

The multiple linear regression analysis showed that serum LH level tended to be negatively associated with WHR ( $\beta-14.325 ; P=0.052)$ in the control group (Table 5). Serum testosterone level was negatively correlated with age $(\beta-0.029 ; P=0 \cdot 010)$ and energy intake $(\beta-0.0001 ; P=0 \cdot 017)$ in the control group. Serum SHBG level was negatively associated with BMI $(\beta-2 \cdot 764 ; P=0.001)$ and fasting insulin level ( $\beta-0.279 ; P=0.022)$ in the control group. Serum testosterone level was negatively associated with age $(\beta-0.279 ; P=0.008)$ and 2 -h insulin level $(\beta-0.279 ; P=0.025)$ in the PCOS group. Serum SHBG level was only negatively associated with BMI $(\beta-3.394 ; P=0.007)$ in the PCOS group, and a correlation $(\beta-6.325 ; \quad P=0.009)$ also existed in the PCOS group with serum testosterone level $\leq 1.7 \mathrm{nmol} / 1$ (Table 6). Serum SHBG level was negatively correlated with macronutrient intake (carbohydrate: $\beta-4404 \cdot 103, \quad P=0.043$; protein: $\beta-4520 \cdot 923, P=0.047$; fat: $\beta-4376.076, P=0.044)$ in the PCOS group with serum testosterone level $>1.7 \mathrm{nmol} / \mathrm{l}$. Consistent with the findings using a simple linear regression model, the results indicated that BMI was a better predictive indicator for serum SHBG level in both the control and

Table 6. Relationships between sex hormones and independent variables including characteristics, metabolic parameters and dietary intake in the polycystic ovary syndrome group $(n 45)$ by serum testosterone level using a multiple linear regression model

(Regression coefficients $(\beta)$ )

\begin{tabular}{|c|c|c|c|c|c|c|c|c|}
\hline & \multicolumn{4}{|c|}{$\begin{array}{l}\text { Testosterone } \leq 1.7 \mathrm{nmol} / \mathrm{l} \\
(n 24)\end{array}$} & \multicolumn{4}{|c|}{$\begin{array}{l}\text { Testosterone }>1.7 \mathrm{nmol} / / \\
(n 21)\end{array}$} \\
\hline & $\begin{array}{l}\text { FSH } \\
(\mathrm{IU} / \mathrm{I})\end{array}$ & $\begin{array}{l}\mathrm{LH} \\
(\mathrm{IU} / \mathrm{I})\end{array}$ & $\begin{array}{l}\text { Testosterone } \\
(\mathrm{nmol} / \mathrm{l})\end{array}$ & $\begin{array}{l}\text { SHBG } \\
(\mathrm{nmol} / \mathrm{l})\end{array}$ & $\begin{array}{l}\mathrm{FSH} \\
(\mathrm{IU} / \mathrm{I})\end{array}$ & $\begin{array}{l}\mathrm{LH} \\
(\mathrm{IU} / \mathrm{I})\end{array}$ & $\begin{array}{l}\text { Testosterone } \\
(\mathrm{nmol} / \mathrm{l})\end{array}$ & $\begin{array}{l}\text { SHBG } \\
(\mathrm{nmol} / \mathrm{l})\end{array}$ \\
\hline \multicolumn{9}{|l|}{ Characteristics } \\
\hline Age (years) & 0.021 & $0 \cdot 156$ & -0.015 & $-1 \cdot 165$ & -0.026 & 0.148 & -0.057 & -0.060 \\
\hline $\operatorname{BMI}\left(\mathrm{kg} / \mathrm{m}^{2}\right)$ & -0.024 & 0.148 & 0.001 & $-6 \cdot 325^{\star \star}$ & -0.021 & -0.150 & -0.017 & $-1 \cdot 141$ \\
\hline WHR & -3.865 & -7.728 & 0.233 & -34.748 & $-29 \cdot 323^{*}$ & 0.284 & 0.287 & $-57 \cdot 814$ \\
\hline \multicolumn{9}{|l|}{ Glucose metabolism } \\
\hline Fasting glucose $(\mathrm{mmol} / \mathrm{l})$ & 0.065 & 0.861 & 0.133 & $9 \cdot 209$ & -0.709 & -0.989 & 0.110 & 9.981 \\
\hline 2-h glucose $(\mathrm{mmol} / \mathrm{l})$ & -0.000 & -0.576 & -0.052 & -1.549 & 0.987 & 0.663 & -0.137 & $-11 \cdot 310$ \\
\hline Fasting insulin $(\mathrm{pmol} / \mathrm{l})$ & 0.039 & -0.033 & -0.004 & 0.015 & 0.004 & 0.059 & -0.005 & 0.021 \\
\hline 2-h insulin (pmol//) & -0.001 & -0.002 & 0.000 & 0.007 & 0.003 & -0.007 & 0.001 & 0.042 \\
\hline \multicolumn{9}{|l|}{ Dietary intake } \\
\hline Energy $(\mathrm{kJ} / \mathrm{d})$ & 0.000 & 0.001 & 0.000 & 0.001 & 0.001 & -0.002 & 0.000 & -0.001 \\
\hline Carbohydrate (\% of energy) & -33.865 & $212 \cdot 144$ & $8 \cdot 776$ & $995 \cdot 705$ & -191.863 & $1533 \cdot 192$ & -17.845 & $-4404 \cdot 103^{*}$ \\
\hline Protein (\% of energy) & $-47 \cdot 164$ & 212.623 & $8 \cdot 772$ & 995.834 & -178.656 & 1620.894 & $-26 \cdot 337$ & $-4520.923^{\star}$ \\
\hline Fat (\% of energy) & $-41 \cdot 226$ & $219 \cdot 103$ & 8.470 & $1035 \cdot 428$ & $-200 \cdot 100$ & $1488 \cdot 223$ & $-15 \cdot 885$ & $-4376.076^{\star}$ \\
\hline
\end{tabular}

FSH, follicle-stimulating hormone; $\mathrm{LH}$, luteinising hormone; SHBG, sex hormone-binding globulin; WHR, waist:hip ratio.

$P<0.05,{ }^{* *} P<0.01$. 
PCOS groups. Serum FSH level was negatively associated with WHR $(\beta-29.323 ; P=0.043)$ in the PCOS group with serum testosterone level $>1.7 \mathrm{nmol} / \mathrm{l}$. However, serum LH level was not significantly associated with characteristics (age, BMI and WHR), glucose metabolic parameters (blood glucose and insulin levels) and dietary intake (energy and macronutrients) in the PCOS group with or without hyperandrogenism.

\section{Discussion}

Similar to the findings of previous studies ${ }^{(5,19,20)}$, women with PCOS had higher BMI than control women. Some of the studies have demonstrated that obesity commonly exists in $30-70 \%$ women with $\operatorname{PCOS}^{(4,6,20-22)}$. However, our data showed that only $13 \%$ women with PCOS had BMI $\geq 27 \mathrm{~kg} / \mathrm{m}^{2}$ and most women with PCOS were non-obese (BMI $23.0(\mathrm{sD} 4.4) \mathrm{kg} / \mathrm{m}^{2}$ ), which is consistent with the study of women with PCOS (BMI 22.7 (SD $4 \cdot 1) \mathrm{kg} / \mathrm{m}^{2}$ ) in southern China ${ }^{(23)}$. Therefore, the prevalence of obesity in women with PCOS is different between Chinese and Caucasian women. Overweight and obesity in women with PCOS exacerbated the severity of hyperandrogenism and impaired the metabolic profiles ${ }^{(24)}$. The present study showed that women with PCOS also had higher waist circumference and WHR than control women, indicating that women with PCOS could have more abdominal fat. In addition, FAI was positively correlated with BMI, waist circumference and HOMA-IR in women with PCOS. Regardless of overweight or obesity, the development of PCOS was strongly associated with weight gain and abdominal fat was correlated with hyperandrogenism and insulin resistance in women with $\operatorname{PCOS}^{(25,26)}$.

The clinical measurements varied due to different ethnic groups, BMI and hormone levels. The PCOS group with the mean age of 32.7 years and the mean BMI of $23.0 \mathrm{~kg} / \mathrm{m}^{2}$ in the present study had higher mean serum FSH $(6.8 v$. $5.0 \mathrm{IU} / \mathrm{l}), \mathrm{LH}(7.3 v \cdot 6 \cdot 2 \mathrm{IU} / \mathrm{l})$, testosterone $(1.8 v \cdot 1.4 \mathrm{nmol} / \mathrm{l})$ and SHBG ( $49 \cdot 4 v \cdot 20 \cdot 4 \mathrm{nmol} / \mathrm{l})$ levels, but lower FAI ( $4.68 v$. 7.38) and HOMA-IR (1.8v.3.3) compared with PCOS patients with a mean age of $27 \cdot 7$ years and a mean BMI of $34.1 \mathrm{~kg} / \mathrm{m}^{2}$ in Bahrain ${ }^{(27)}$. The PCOS group in the present study had a higher mean FSH level $(6 \cdot 8 v$. $5 \cdot 0 \mathrm{IU} / 1)$, but lower LH level $(7 \cdot 3 v .13 \cdot 0 \mathrm{IU} / \mathrm{l})$, LH:FSH $(1 \cdot 1 v \cdot 2 \cdot 6)$, testosterone $(1 \cdot 8 v$. $3.2 \mathrm{nmol} / \mathrm{l})$ and FAI (4.68 v. 11.3) compared with PCOS patients with a mean age of $32 \cdot 2$ years and a mean BMI of $29.9 \mathrm{~kg} / \mathrm{m}^{2}$ in the $\mathrm{UK}^{(28)}$. The PCOS group in the present study had lower BMI and FAI compared with the previous studies $^{(27,28)}$, which is consistent with our findings that FAI was reduced with decreased BMI in women with PCOS.

The present study demonstrated that women with PCOS had higher serum LH, LH:FSH, serum testosterone and FAI and lower serum SHBG levels, which is consistent with hormonal changes for the diagnosis of PCOS. A previous study found that obese women with PCOS had significantly higher serum total testosterone levels than non-obese women with $\operatorname{PCOS}^{(29)}$. However, the present study showed that in the PCOS group serum testosterone level was not significantly different in the group with $\mathrm{BMI} \geq 22 \mathrm{~kg} / \mathrm{m}^{2}$ compared with the group with BMI $<22 \mathrm{~kg} / \mathrm{m}^{2}$, which is consistent with our findings that serum testosterone level was associated with neither BMI nor waist circumference in women with PCOS. The different findings could be attributed to different proportions of overweight and obese women with PCOS and degree of hyperandrogenism.

The changes in hormones including serum SHBG and FAI were greater in women with PCOS with BMI $\geq 22 \mathrm{~kg} / \mathrm{m}^{2}$ compared with those with BMI $<22 \mathrm{~kg} / \mathrm{m}^{2}$, suggesting that hormonal changes are strongly associated with BMI. In agreement with a previous study ${ }^{(30)}$, serum SHBG level was negatively associated with body weight, BMI and waist circumference, while FAI was positively associated with these parameters mentioned above, in women with PCOS. In addition, as compared with women with PCOS who had BMI $<22 \mathrm{~kg} / \mathrm{m}^{2}$, those with BMI $\geq 22 \mathrm{~kg} / \mathrm{m}^{2}$ had significantly increased 2-h serum glucose, fasting insulin, 2-h serum insulin and HOMA-IR which were positively correlated with body weight, BMI and waist circumference. Women with PCOS and impaired glucose metabolism had higher incidences of glucose intolerance and type 2 diabetes mellitus ${ }^{(5,31)}$. Therefore, it is reasonable to suspect that increased BMI in women with PCOS impairs insulin sensitivity which can further influence hyperandrogenism. Insulin insensitivity was found to possibly contribute to hyperandrogenism ${ }^{(32)}$. Elevated insulin level and HOMA-IR in women with PCOS who had higher BMI values in the present study could contribute to the increases in serum testosterone and FAI. Increased insulin level also possibly leads to lower serum SHBG. There was a strong negative correlation between fasting insulin level and serum SHBG in obese hyperandrogenic women ${ }^{(33)}$ and women with $\operatorname{PCOS}^{(34)}$. Martens et al. ${ }^{(35)}$ hypothesised that serine hyperphosphorylation of cytochrome P450c17 increasing 17,20-lyase activity and androgen production may cause hyperandrogenism and serine hyperphosphorylation of insulin receptor- $\beta$ inhibiting its tyrosine phosphorylation may cause insulin resistance in women with PCOS.

A previous study in the UK reported that women with PCOS who had a mean BMI of $27 \cdot 4 \mathrm{~kg} / \mathrm{m}^{2}$ consumed $38 \%$ of energy intake from fat ${ }^{(36)}$, which was higher than that (31\%) in those with the mean BMI of $23.0 \mathrm{~kg} / \mathrm{m}^{2}$ in the present study. Similar to the present results for macronutrient intake (18, 31 and 51\% of energy intake from protein, fat and carbohydrate, respectively) in women with PCOS, a previous study in Italy showed that habitual intake for protein, fat and carbohydrate was 16, 33 and $52 \%$ of energy intake, which was a similar distribution between women with PCOS and hypothalamic amenorrhea $^{(37)}$. The consumption of energy and macronutrients was not significantly different between women with PCOS and age-, race- and BMI-matched healthy women in the study by Douglas et al. ${ }^{(38)}$. However, women with PCOS consumed more high-glycaemic index foods, such as white bread (7.9 (SD 4.4) v. 5.5 (SD 2.9) servings over $4 \mathrm{~d} ; P<0.05$ ) and fried potatoes (1.0 (SD 1.5) v. 0.4 (SD 0.7) servings over $4 \mathrm{~d} ; P=0.07)$ than did the healthy control group ${ }^{(38)}$. The present study found that women with PCOS had lower energy and carbohydrate intake but higher fat intake than infertile women without PCOS. The different findings between the 
study by Douglas et $a l .{ }^{(38)}$ and the present study could be due to different anthropometric characteristics (race: mostly white Americans $v$. Chinese, age: $28.9 v$. 32.7-34.7 years and BMI: $29 \cdot 1-29 \cdot 7$ v. $21 \cdot 3-23 \cdot 0 \mathrm{~kg} / \mathrm{m}^{2}$ ), dietary habits (different cuisines), biochemical data (fasting glucose and insulin levels, HOMA-IR: $2.9-4.6 \quad v \cdot 1 \cdot 37-1 \cdot 81$ ) and comparing subjects (healthy $v$. non-PCOS-related infertile women). However, the present study only used 3-d dietary record to assess dietary intake, but did not use a FFQ, particularly for sweets and high-glycaemic index foods, which could be further studied.

Compared with the results of the Nutrition and Health Survey in Taiwan 2005-2008, the average energy intake $(1617 \mathrm{kcal} / \mathrm{d} ; 6766 \mathrm{~kJ} / \mathrm{d})$ in the control group with the mean age of 34.7 years was very similar to that $(1671 \mathrm{kcal} / \mathrm{d}$; $6991 \mathrm{~kJ} / \mathrm{d}$ ) in healthy women aged $31-64$ years $^{(39)}$. The average energy intake in both the control and PCOS groups (1617 $v .1508 \mathrm{kcal} / \mathrm{d} ; 6766 v .6311 \mathrm{~kJ} / \mathrm{d}$ ) fell within the current dietary reference intake in Taiwan for energy $(1450-2100 \mathrm{kcal} / \mathrm{d}$; $6070-8790 \mathrm{~kJ} / \mathrm{d}$ ) in women aged $31-50$ years $^{(40)}$. The average intake $(\mathrm{g} / \mathrm{d})$ for carbohydrate, fat and protein was 102, 88 and $96 \%$ in the control group (222, 51 and $68 \mathrm{~g} / \mathrm{d}$ ) compared with those $(218,58$ and $71 \mathrm{~g} / \mathrm{d})$ in healthy women aged 31 to 64 years in Taiwan ${ }^{(39)}$, while the PCOS group had 88, 91 and $94 \%$ of the average intake for carbohydrate, fat and protein (191, 53 and $67 \mathrm{~g} / \mathrm{d})$. However, energy intake from carbohydrate, fat and protein in women with PCOS $(51.5,30 \cdot 8$ and $17 \cdot 6 \%)$ was close to those $(51.8,31.2$ and $17.0 \%)$ in healthy women aged 31-64 years in Taiwan.

Weight loss through energy restriction has been demonstrated to positively affect metabolic and hormonal profiles in women with $\operatorname{PCOS}^{(24)}$. The present study revealed that women with PCOS had lower intakes of energy, carbohydrate and percentage of energy from carbohydrate but a higher percentage of energy from fat. However, women with PCOS had higher BMI and waist circumference. This is probably due to decreased energy intake for weight loss in non-obese women with PCOS and higher BMI. However, the measurement error of using dietary records for dietary assessment cannot be ruled out, although the 3-d dietary record reflects current dietary intake more accurately and specifically than dietary recall and FFQ. Dietary composition and energy distribution from macronutrients could also contribute to higher BMI in women with PCOS. However, in agreement with the present study, daily intake of energy and macronutrients has not been found to be associated with insulin resistance ${ }^{(38,41)}$. Other nutrients or food components, such as dietary fibre and antioxidants, could cause metabolic and hormonal changes in women with PCOS. Protein intake was negatively correlated with fasting insulin level and HOMA-IR in the control group but not in women with PCOS, indicating that there could be various relationships between dietary intake and glucose metabolic parameters in the control and PCOS groups due to different anthropometric, metabolic and nutritional status.

Due to the different proportions of overweight and obese women with PCOS and degree of hyperandrogenism between the present study and others carried out in Europid populations, generalisation of the present results is considered to be limited for those non-obese women with PCOS. In conclusion, women with PCOS have lower serum SHBG levels negatively correlated with HOMA-IR and higher FAI positively correlated with HOMA-IR compared with those with non-PCOS-related infertility. Women with PCOS have lower energy and carbohydrate intakes but higher fat intakes, and macronutrient intake is only negatively associated with serum SHBG level in the PCOS group with hyperandrogenism. To minimise the influence of BMI and hyperandrogenism, the comparisons between non-obese and obese women with PCOS and between those with and without hyperandrogenism should be studied further to investigate whether dietary intake or habit is associated with the morbidity of PCOS. In addition, only 3-d dietary records were used for dietary intake evaluation in the present study; 7-d weighed food diaries could be considered for the assessment of dietary habit and under-reporting in women with PCOS in a future study.

\section{Acknowledgements}

This research received no specific grant from any funding agency in the public, commercial or not-for-profit sectors. Y.-H. T. and T.-W. W. participated in data collection, biochemical and statistical analysis of data and preparation of the manuscript. H.-J. W. participated in the concept and design of the study, recruitment and screening of the subjects, interpretation of data and revision of the manuscript. C.-Y. H., H.-J. H. and W.-H. C. participated in statistical analysis and interpretation of data. R. Y. and C.-M. L. participated in data collection and subject screening. J. C.-J. C. participated in the concept and design of the study, interpretation of data and critical revision of the manuscript. None of the authors has declared a conflict of interest.

\section{References}

1. Rotterdam ESHRE/ASRM-Sponsored PCOS Consensus Workshop Group (2004) Revised 2003 consensus on diagnostic criteria and long-term health risks related to polycystic ovary syndrome. Fertil Steril 81, 19-25.

2. Knochenhauer ES, Key TJ, Kahsar-Miller M, et al. (1998) Prevalence of the polycystic ovary syndrome in unselected black and white women of the southeastern United States: a prospective study. J Clin Endocrinol Metab 83, 3078-3082.

3. March WA, Moore VM, Willson KJ, et al. (2010) The prevalence of polycystic ovary syndrome in a community sample assessed under contrasting diagnostic criteria. Hum Reprod 25, 544-551.

4. Azziz R, Woods KS, Reyna R, et al. (2004) The prevalence and features of the polycystic ovary syndrome in an unselected population. J Clin Endocrinol Metab 89, 2745-2749.

5. Legro RS, Kunselman AR, Dodson WC, et al. (1999) Prevalence and predictors of risk for type 2 diabetes mellitus and impaired glucose tolerance in polycystic ovary syndrome: a prospective, controlled study in 254 affected women. J Clin Endocrinol Metab 84, 165-169.

6. Vrbikova J \& Hainer V (2009) Obesity and polycystic ovary syndrome. Obes Facts 2, 26-35.

7. Remsberg KE, Talbott EO, Zborowski JV, et al. (2002) Evidence for competing effects of body mass, hyperinsulinemia, insulin resistance, and androgens on leptin levels among 
lean, overweight, and obese women with polycystic ovary syndrome. Fertil Steril 78, 479-486.

8. Hahn S, Tan S, Elsenbruch S, et al. (2005) Clinical and biochemical characterization of women with polycystic ovary syndrome in North Rhine-Westphalia. Horm Metab Res 37, 438-444.

9. Ni RM, Mo Y, Chen X, et al. (2009) Low prevalence of the metabolic syndrome but high occurrence of various metabolic disorders in Chinese women with polycystic ovary syndrome. Eur J Endocrinol 161, 411-418.

10. Dunaif A (1997) Insulin resistance and the polycystic ovary syndrome: mechanism and implications for pathogenesis. Endocr Rev 18, 774-800.

11. Ovalle F \& Azziz R (2002) Insulin resistance, polycystic ovary syndrome, and type 2 diabetes mellitus. Fertil Steril 77, 1095-1105.

12. Chang RJ, Nakamura RM, Judd HL, et al. (1983) Insulin resistance in nonobese patients with polycystic ovarian disease. J Clin Endocrinol Metab 57, 356-359.

13. Galluzzo A, Amato MC \& Giordano C (2008) Insulin resistance and polycystic ovary syndrome. Nutr Metab Cardio Dis 18, 511-518.

14. Thomson RL, Buckley JD \& Brinkworth GD (2011) Exercise for the treatment and management of overweight women with polycystic ovary syndrome: a review of the literature. Obes Rev 12, e202-e210.

15. Moran LJ, Noakes M, Clifton PM, et al. (2006) Short-term meal replacements followed by dietary macronutrient restriction enhance weight loss in polycystic ovary syndrome. Am J Clin Nutr 84, 77-87.

16. Marsh KA, Steinbeck KS, Atkinson FS, et al. (2010) Effect of a low glycemic index compared with a conventional healthy diet on polycystic ovary syndrome. Am J Clin Nutr 92, 83-92.

17. Matthews DR, Hosker JP, Rudenski AS, et al. (1985) Homeostasis model assessment: insulin resistance and $\beta$-cell function from fasting plasma glucose and insulin concentrations in man. Diabetologia 28, 412-419.

18. Hatch R, Rosenfield RL, Kim MH, et al. (1981) Hirsutism: implications, etiology, and management. Am J Obstet Gynecol 140, 815-830.

19. Lee H, Oh JY, Sung YA, et al. (2009) The prevalence and risk factors for glucose intolerance in young Korean women with polycystic ovary syndrome. Endocrine 36, 326-332.

20. Cupisti S, Kajaia N, Dittrich R, et al. (2008) Body mass index and ovarian function are associated with endocrine and metabolic abnormalities in women with hyperandrogenic syndrome. Eur J Endocrinol 158, 711-719.

21. Carmina E, Legro RS, Stamets K, et al. (2003) Difference in body weight between American and Italian women with polycystic ovary syndrome: influence of the diet. Hum Reprod 18, 2289-2293.

22. Kelestimur F, Unluhizarci K, Baybuga H, et al. (2006) Prevalence of polycystic ovarian changes and polycystic ovary syndrome in premenopausal women with treated type 2 diabetes mellitus. Fertil Steril 86, 405-410.

23. Chen X, Yang D, Mo Y, et al. (2008) Prevalence of polycystic ovary syndrome in unselected women from southern China. Eur J Obstet Gynecol Reprod Biol 139, 59-64.

24. O'Connor A, Gibney J \& Roche HM (2010) Metabolic and hormonal aspects of polycystic ovary syndrome: the impact of diet. Proc Nutr Soc 69, 628-635.
25. Barber TM, McCarthy MI, Wass JAH, et al. (2006) Obesity and polycystic ovary syndrome. Clin Endocrinol 65, 137-145.

26. Diamanti-Kandarakis E (2007) Role of obesity and adiposity in polycystic ovary syndrome. Int J Obes (Lond) 31, S8-S13.

27. Golbahar J, Al-Ayadhi M, Das NM, et al. (2012) Sensitive and specific markers for insulin resistance, hyperandrogenemia, and inappropriate gonadotrophin secretion in women with polycystic ovary syndrome: a case-control study from Bahrain. Int J Women's Health 4, 201-206.

28. Nardo LG, Buckett WM, White D, et al. (2002) Threedimensional assessment of ultrasound features in women with clomiphene citrate-resistant polycystic ovarian syndrome (PCOS): ovarian stromal volume does not correlate with biochemical indices. Hum Reprod 17, 1052-1055.

29. Liou TH, Tang JH, Hsieh CH, et al. (2009) Clinical and biochemical presentation of polycystic ovary syndrome among obese and nonobese women. Fertil Steril 92, 1960-1965.

30. Ducluzeau PH, Cousin P, Malvoisin E, et al. (2003) Glucoseto-insulin ratio rather than sex hormone-binding globulin and adiponectin levels is the best predictor of insulin resistance in nonobese women with polycystic ovary syndrome. J Clin Endocrinol Metab 88, 3626-3631.

31. Wei HJ, Young R, Kuo IL, et al. (2009) Prevalence of insulin resistance and determination of risk factors for glucose intolerance in polycystic ovary syndrome: a cross-sectional study of Chinese infertility patients. Fertil Steril 91, 1864-1868.

32. Legro RS, Castracane VD \& Kauffman RP (2004) Detecting insulin resistance in polycystic ovary syndrome: purposes and pitfalls. Obstet Gynecol Surv 59, 141-154.

33. Pugeat M, Crave JC, Elmidani M, et al. (1991) Pathophysiology of sex hormone binding globulin (SHBG): relation to insulin. J Steroid Biochem Mol Biol 40, 841-849.

34. Jayagopal V, Kilpatrick ES, Jennings PE, et al. (2003) The biological variation of testosterone and sex hormone-binding globulin (SHBG) in polycystic ovarian syndrome: implications for SHBG as a surrogate marker of insulin resistance. J Clin Endocrinol Metab 88, 1528-1533.

35. Martens JW, Geller DH, Arlt W, et al. (2000) Enzymatic activities of $\mathrm{P} 450 \mathrm{c} 17$ stably expressed in fibroblasts from patients with the polycystic ovary syndrome. J Clin Endocrinol Metab 85, 4338-4346.

36. Barr S, Hart K, Reeves S, et al. (2011) Habitual dietary intake, eating pattern and physical activity of women with polycystic ovary syndrome. Eur J Clin Nutr 65, 1126-1132.

37. Colombo O, Pinelli G, Comelli M, et al. (2009) Dietary intakes in infertile women a pilot study. Nutr J 8, 53.

38. Douglas CC, Norris LE, Oster RA, et al. (2006) Difference in dietary intake between women with polycystic ovary syndrome and healthy controls. Fertil Steril 86, 411-417.

39. Wu SJ, Pan WH, Yeh NH, et al. (2011) Trends in nutrient and dietary intake among adults and the elderly: from NAHSIT 1993-1996 to 2005-2008. Asia Pac J Clin Nutr 20, 251-265.

40. Wang GS (editor) (2012) Dietary Reference Intakes. 7th ed. Taipei, Taiwan: Food and Drug Administration, Department of Health, Taiwan, R.O.C.

41. Toscani MK, Mario FM, Radavelli-Bagatini S, et al. (2011) Insulin resistance is not strictly associated with energy intake or dietary macronutrient composition in women with polycystic ovary syndrome. Nutr Res 31, 97-103. 